\title{
A Rare Cause of Chest Pain: Disseminated Elemental Mercury Microthromboembolism
}

\author{
Brandon Allen, Bobby Desai, Lars K. Beattie \\ Department of Emergency Medicine, Gainesville, FL, USA \\ Email: brandonrallen@ufl.edu, bdesai@ufl.edu, lars.beattie@ufl.edu \\ Received 30 November 2015; accepted 19 January 2016; published 22 January 2016 \\ Copyright (C) 2016 by authors and Scientific Research Publishing Inc. \\ This work is licensed under the Creative Commons Attribution International License (CC BY). \\ http://creativecommons.org/licenses/by/4.0/ \\ cC) (i) Open Access
}

\begin{abstract}
Background: The clinical manifestations of mercury poisoning vary based on chemical form, dose, and route of administration. In the medical field, many of the cases of mercury exposure have been in mining agriculture, or chemical industrial workers via inhalation. Aim: To discuss the diagnosis of parenteral mercury poisoning with radiographic identification and potential sequelae. Case Presentation: We present the case of a young male with a chief complaint of chest pain and intravenous mercury administration found to be intentional as a suicide attempt. The chest radiographs lead to a wide differential diagnosis in a hemodynamically stable patient. Conclusion: While ingestion rarely causes toxicity due to the poor absorption of mercury through the gastrointestinal tract, intravenous elemental mercury can range from producing minimal symptoms to being severely toxic.
\end{abstract}

\section{Keywords}

Mercury Toxicity, Chest Pain, Microthromboembolism

\section{Introduction}

Mercury has been used widely for many centuries in various treatment modalities and medical instruments. Chronic exposure to vapor of mercury has been reported to result in neurologic symptoms like weakness, ataxia, and tremors. Acute exposure is more dramatic and can be fatal [1] [2].

Toxicity with metallic mercury injection, acquired both accidentally, and following attempted suicide is extremely rare. There have been a few published cases of intravenous metallic mercury injection over the past 30 years most commonly found in patients who are younger males. 


\section{Case Report}

\subsection{Emergency Department Evaluation}

A 31-year-old male with the past medical history of spontaneous pneumothorax and hepatitis $C$ presented to the Emergency Department with a complaint of severe, stabbing left-sided chest pain which began abruptly 4 hours prior to arrival and was worse with deep inspiration throughout the chest. He also reported that this pain was different from his previous pneumothorax chest pain. He acknowledged a non-productive cough, but denied fevers, chills or rigors. His only medication was ibuprofen $800 \mathrm{mg}$ every six hours as needed.

The pertinent social history included that the patient was an active full pack per daysmoker with frequent use of marijuana. He denied IV drug use and worked as a part-time mechanic whose duties consisted of welding and grinding metal. He reported no protective equipment during these activities.

At presentation to the emergency department his initial vital signs and physical exam showed: temperature 37.9 degrees Celsius, pulse rate 96 beats per minute, blood pressure 124/83 mmHg, respiration rate 24 breaths per minute, and pulse oximetry $99 \%$ on room air. He was in apparent distress, writhing in pain, tachypneic, and he appeared disheveled and unkempt. His chest wall was non-tender to palpation, bilateral normal percussion, vocal fremitus, symmetrically clear breath sounds without crepitation or audible wheezing. His heart sounds were normal without murmur, rub or gallop. Besides multiple excoriations and a few pustules in accessible areas of his body, the rest of the patient's physical exam was normal and unrevealing.

Initial laboratory data revealed white blood cell count of 12.1 thousand/cu mm with $62.7 \%$ neutrophils and no bandemia. The cardiac biomarkers, basic metabolic panel, coagulation studies and urinalysis were all normal. No inclusion criteria were met based on the Pulmonary Embolism Rule-Out Criteria (PERC) to warrant a d-dimer in this patient [3].

The radiograph from his most recent emergency department discharge (Figure 1 and Figure 2) showed no acute cardiopulmonary disease and a two-view chest radiograph was performed (Figure 3 and Figure 4) which showed scattered radiopaque material between both lungs consistent with inhalation of radiopaque material. He was then admitted to the hospital.

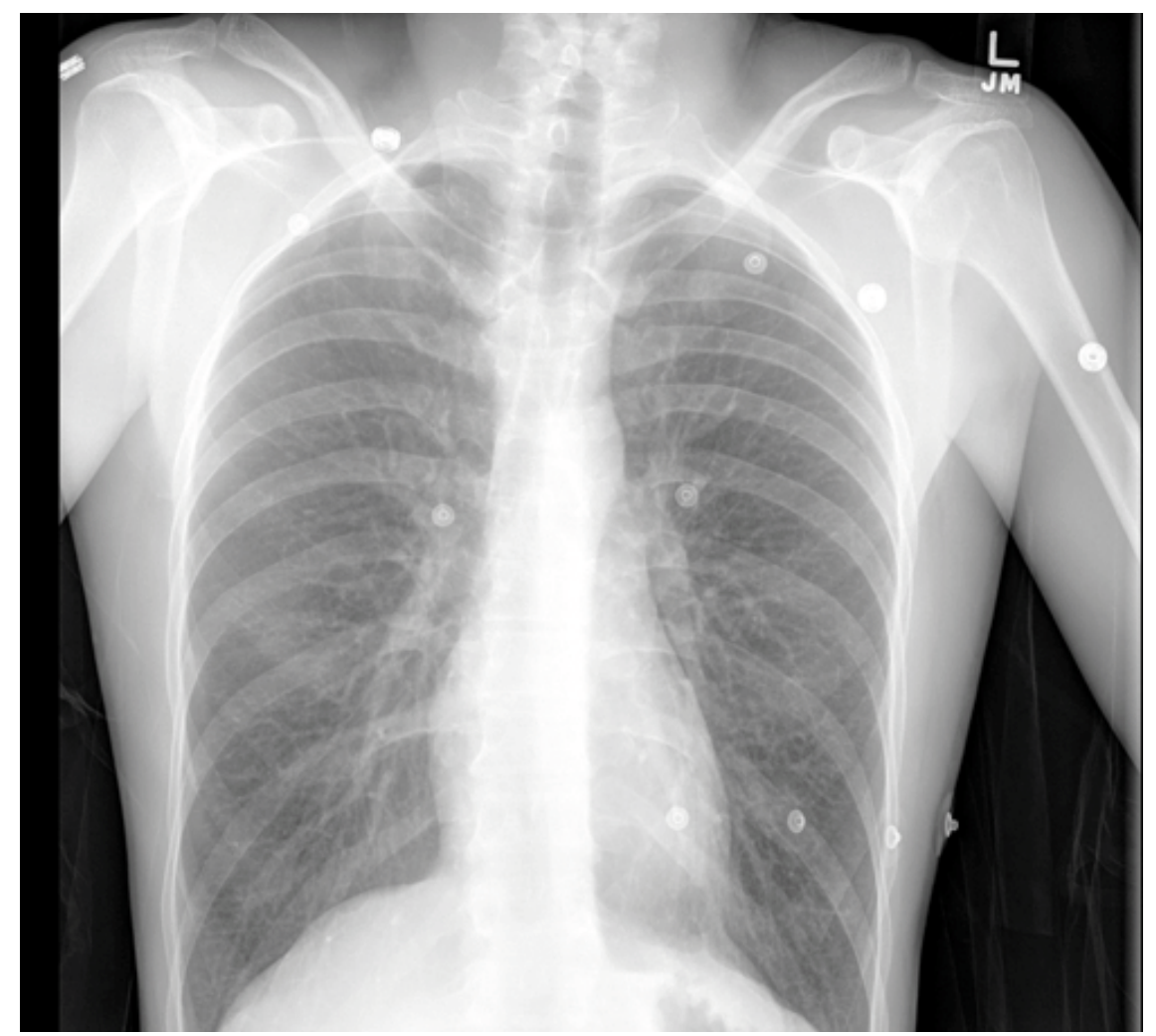

Figure 1. Posterior/anterior chest radiograph. Interpretation: no acute cardiopulmonary disease. 


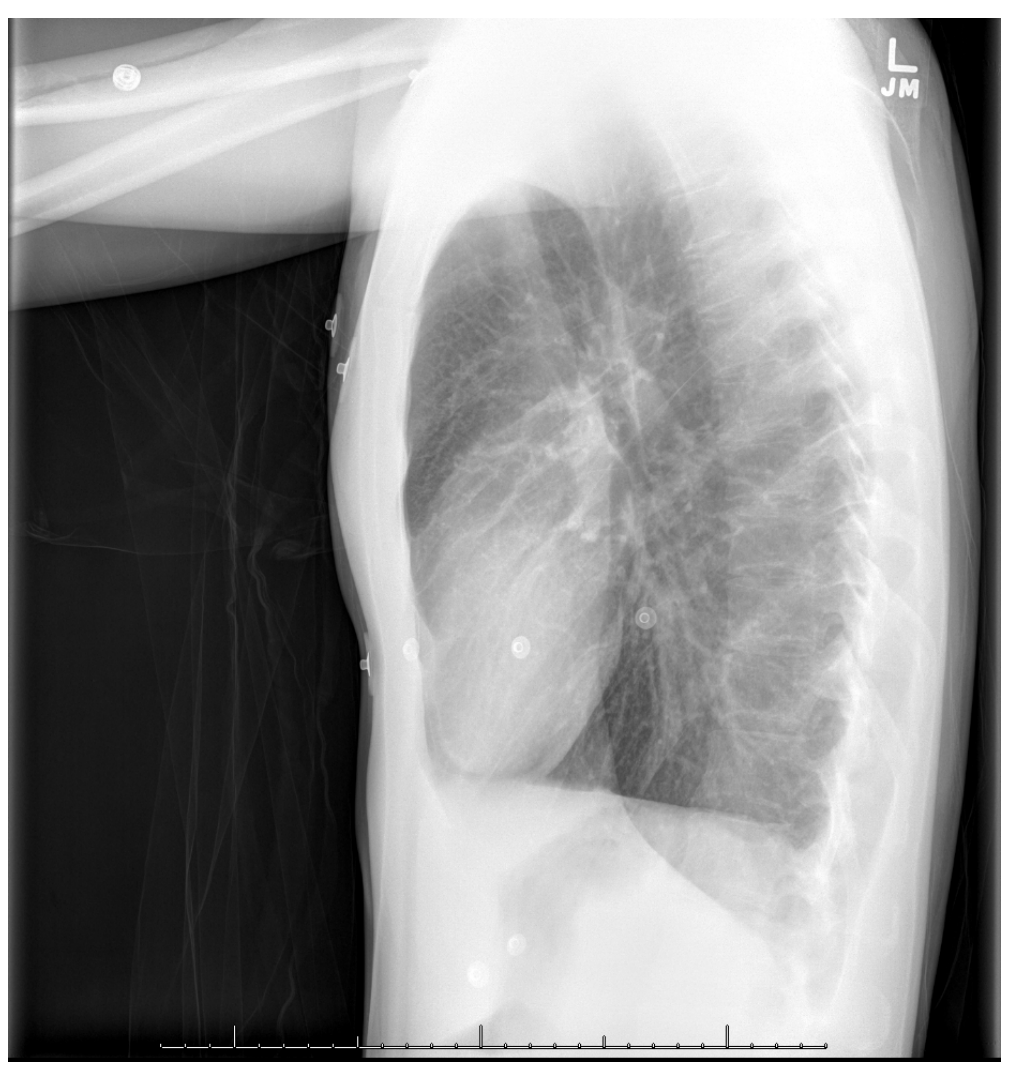

Figure 2. Lateral chest radiograph. Interpretation: no acute cardiopulmonary disease.

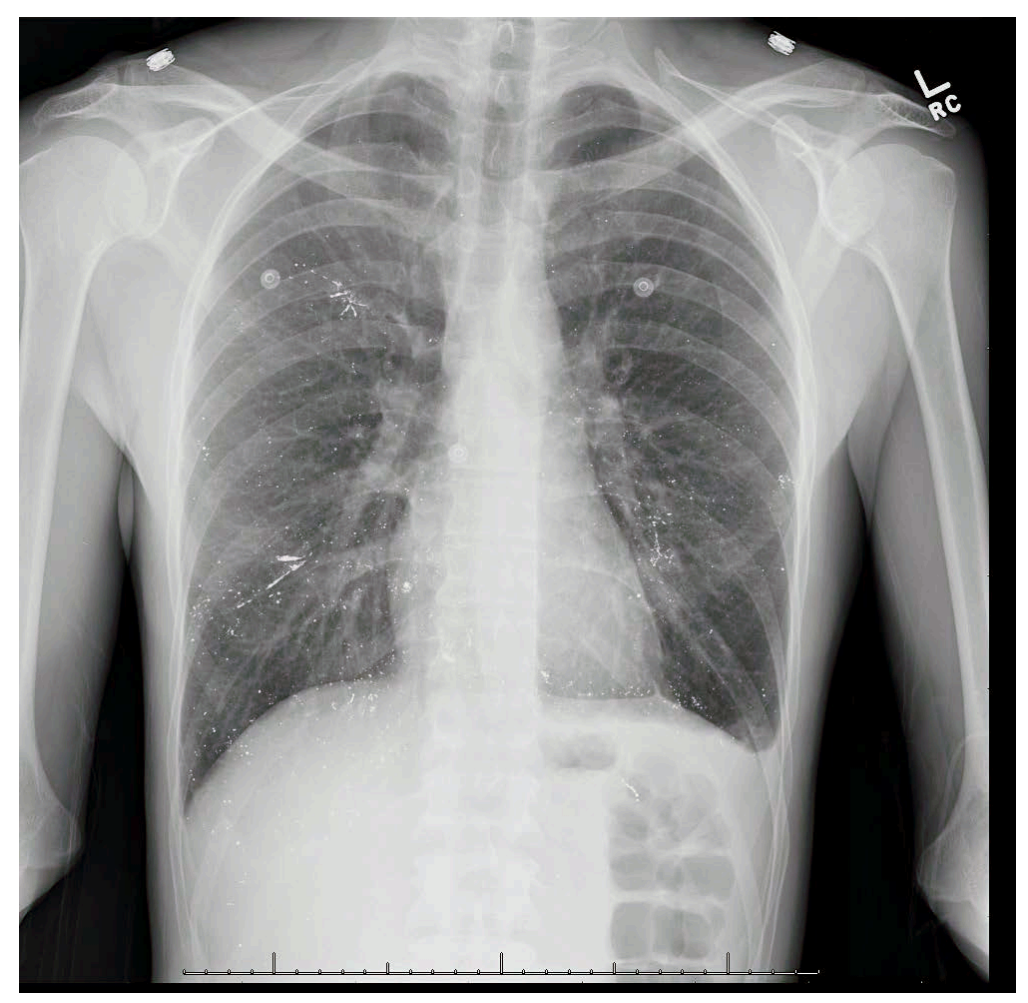

Figure 3. Posterior/anterior chest radiograph. Preliminary radiology interpretation: scattered radiopaque material between both lungs consistent with inhalation of radiopaque material. 


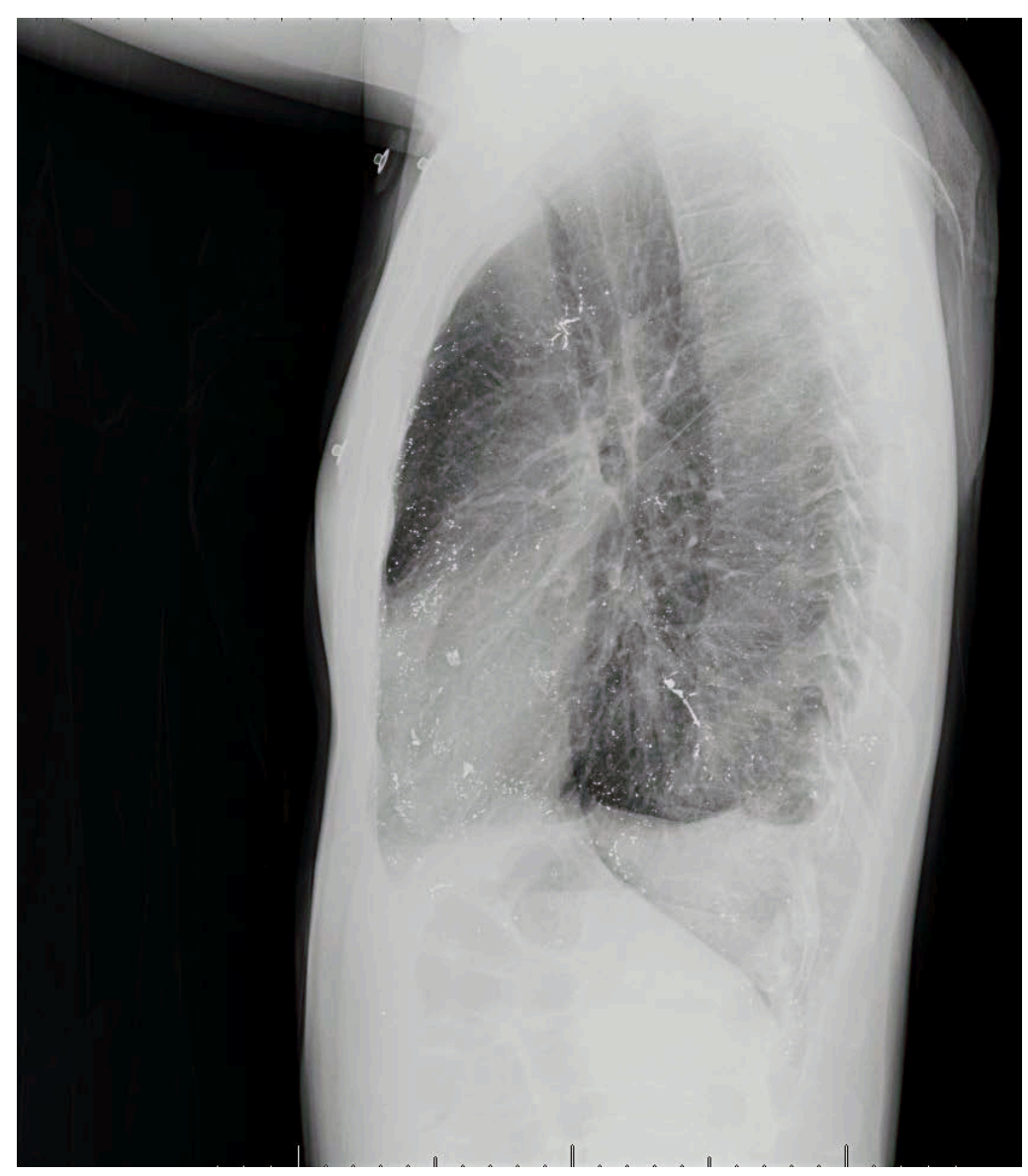

Figure 4. Lateral chest radiograph. Preliminary radiology interpretation: Scattered radiopaque material between both lungs consistent with inhalation of radiopaque material.

\subsection{Hospital Course}

During his hospital stay patient continued to have severe pleuritic chest pain with moderate relief from parenteral narcotics. The patient underwent a CT scan (Figures 5-9) to evaluate for thromboembolism and innumerable metallic foreign bodies were found scattered throughout each hemithorax, right ventricle and the liver which were not present in a prior study from one week before his hospital admission.

Considering his presentation and wide spread metallic microthromboemboli outside the lung tissues, inhalation of metallic objects due to unprotected welding was deemed unlikely. An intravenous metallic injection was suspected, and the Poison Control Center was contacted. A heavy metal urine toxicology collection was ordered. The following morning after urine collection, the patient's chest pain had improved and he requested hospital discharge. He was advised to wait for urine toxicology results in order to guide therapy, but he consequently signed out against medical advice. The results returned three days later revealing urine mercury $54 \mathrm{ug} / \mathrm{L}$ (Reference range 0 - 10), urine mercury ug/day 104 (Reference range 0 - 15 ug/day) and urine mercury ug/g creatinine was 79.4 (Reference range $<=35$ ) [4].

Once the toxicology report had resulted, the patient was appropriate for chelation therapy to remove the mercury as the primary therapeutic intervention. He was unable to be contacted despite numerous attempts and a law enforcement visit to the patient's last known address. Based upon historical chart review, the patient has returned to the ED with the complaint of chest pain. However, he has not shown evidence or sequelae of toxicity from his intravenous mercury exposure.

\subsection{Diagnosis}

Disseminated elemental mercury microthromboembolism. 


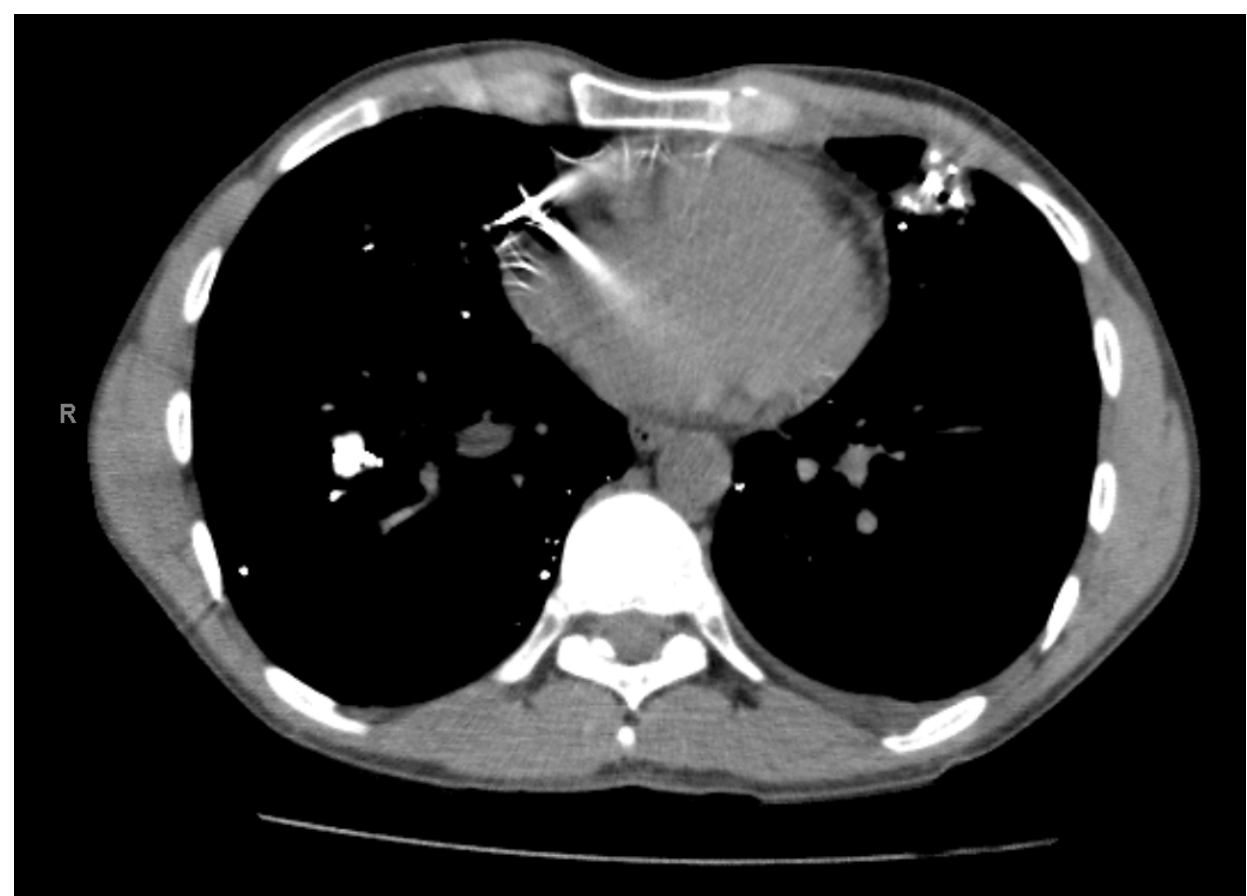

Figure 5. CT scan of the chest. Interpretation: multiple metallic foreign bodies found scattered throughout each hemithorax and right ventricle.

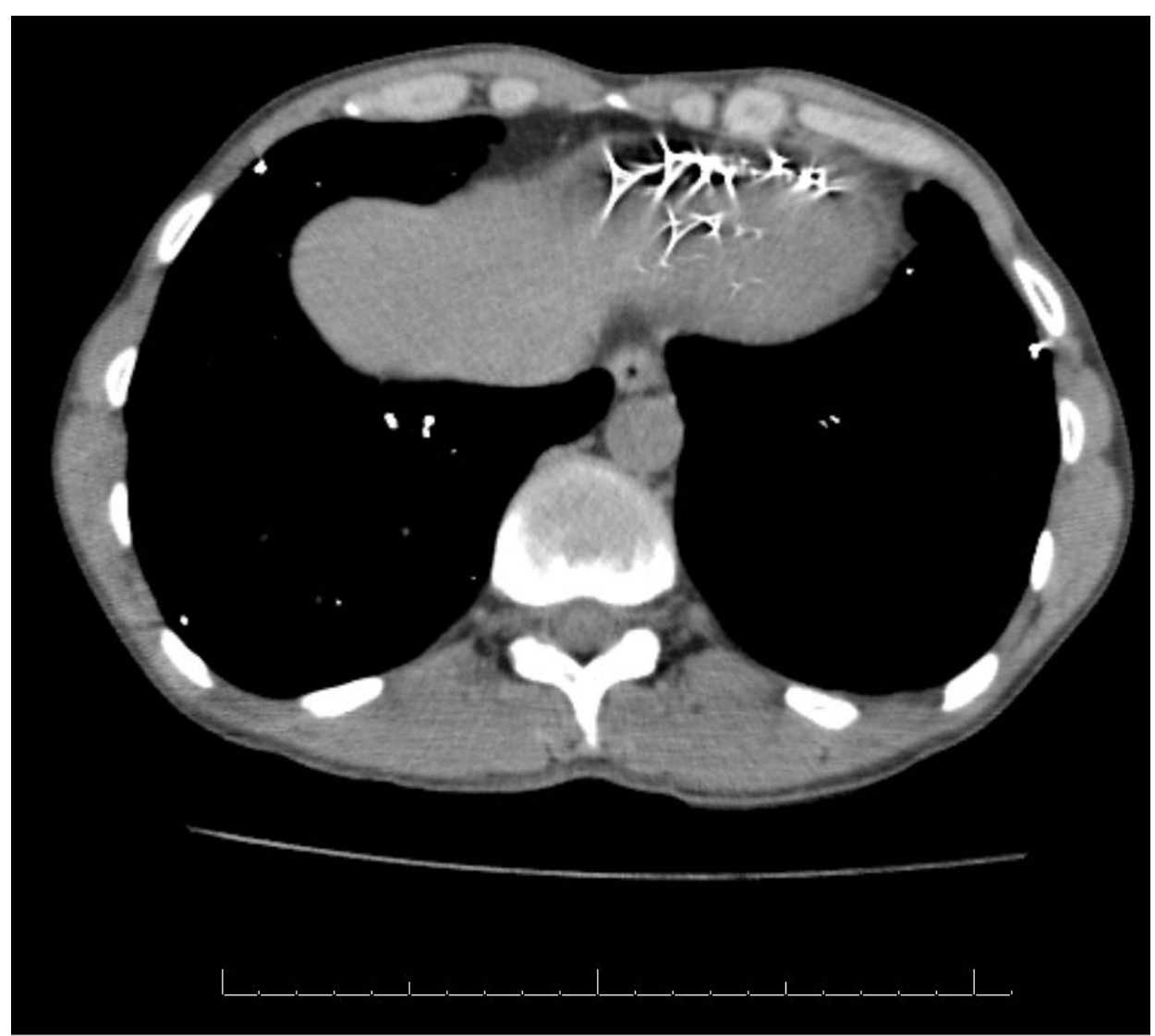

Figure 6. CT scan of the chest. Interpretation: multiple metallic foreign bodies found scattered throughout each hemithorax and liver. 


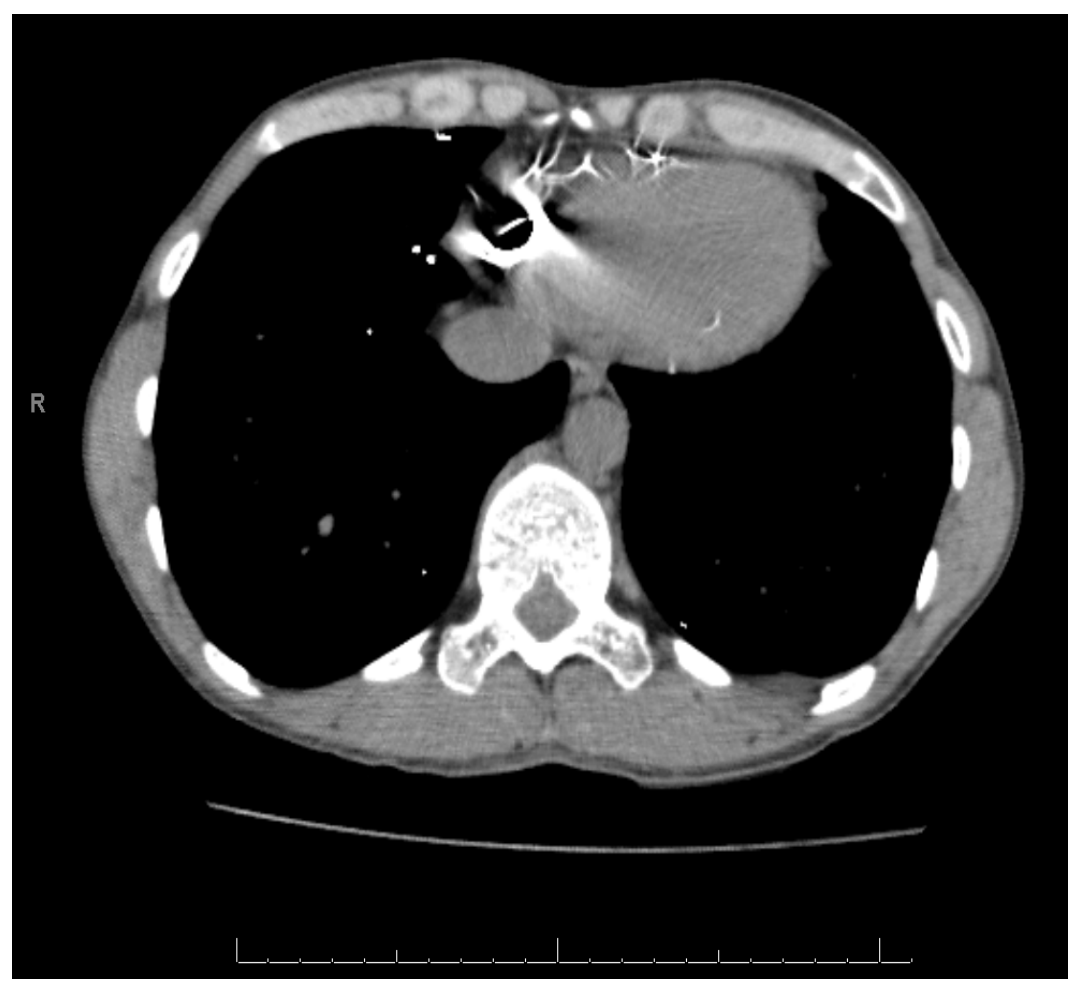

Figure 7. CT scan of the chest. Interpretation: multiple metallic foreign bodies found scattered throughout each hemithorax and right ventricle.

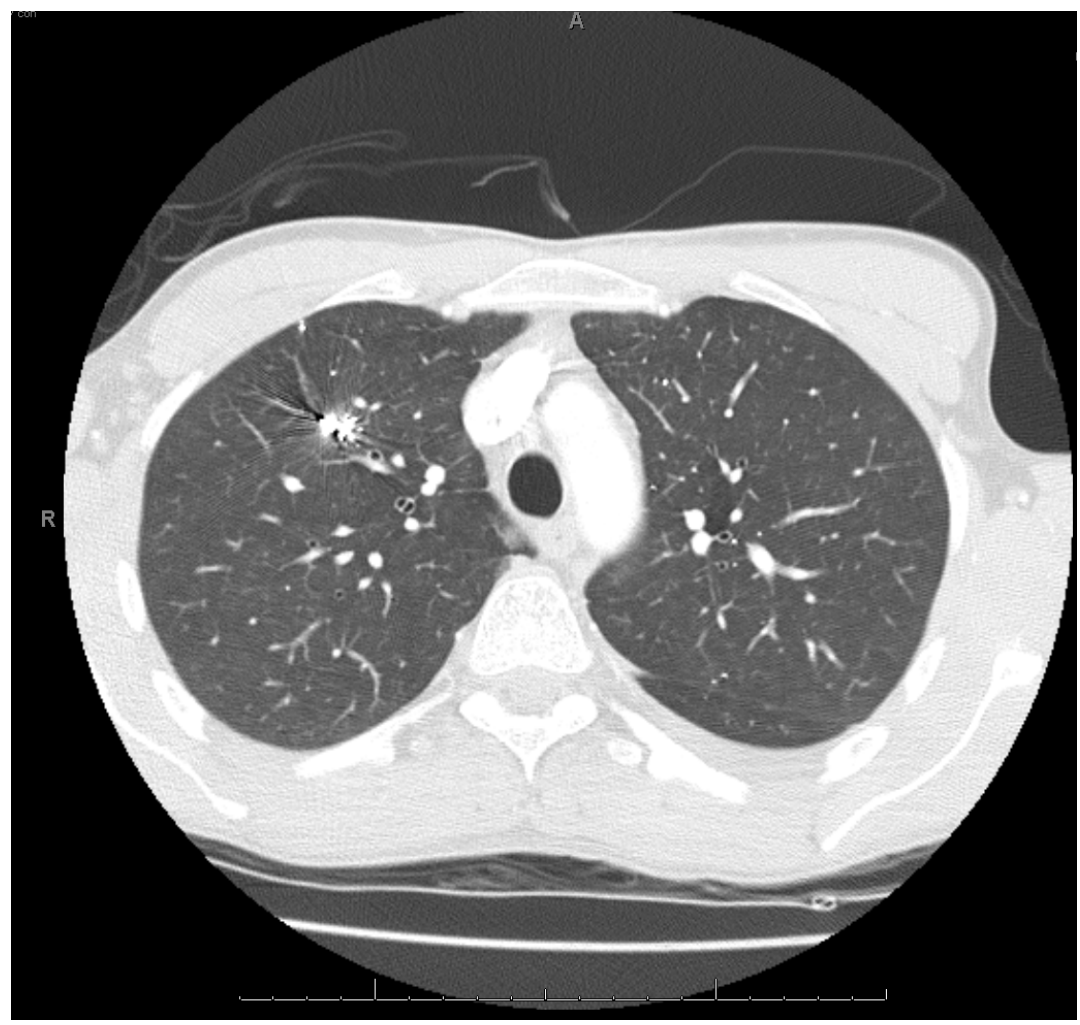

Figure 8. CT scan of the chest. Interpretation: multiple metallic foreign bodies found scattered throughout each hemithorax and bilateral lung fields. 


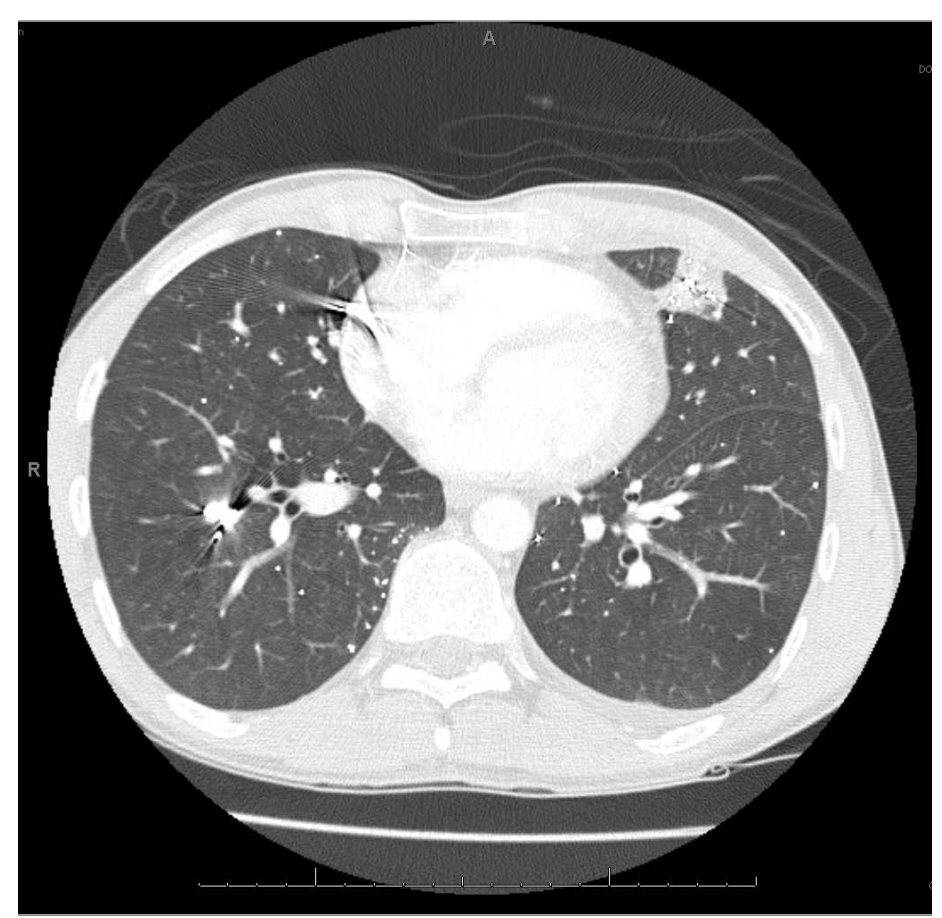

Figure 9. CT scan of the chest. Interpretation: multiple metallic foreign bodies found scattered throughout each hemithorax, bilateral lung fields, and right ventricle.

\section{Discussion}

While some patients with disseminated elemental mercury microthromboembolism have reportedly presented with fever, cough, GI symptoms, gingivitis, intention tremor, and/or bizarre behavior, this patient was unique as he only had symptoms of severe chest pain [5].

Because elemental mercury is radiopaque, it can be easily detected radiographically [6]. The chest radiograph usually shows tiny metallic opacities throughout the lung fields. A CT scan of the chest, abdomen and pelvis shows the full extent of mercury deposits throughout various organs [7]. The degree of mercury toxicity can further be evaluated by obtaining blood and urine mercury levels. Blood concentrations of $>2$ microgram/dL and urinary concentrations of $>20$ microgram/dL indicate toxicity [4]. Our patient's mercury level was $54 \mathrm{ug} / \mathrm{L}$.

Management of metallic mercury toxicity depends on severity of the illness, which in turn depends on the quantity injected, and the extent of tissue distribution. Embolization to the lung is seen in most cases. In rare cases, the mercury passes throughout the pulmonary vascular bed and may enter into systemic circulation resulting in liver disturbances, acute renal failure, anemia, various GI and neurologic abnormalities. Based on the mercury level, DMSA (Dimercaptosuccinic acid) or British Anti-Lewisite (BAL) may be given as a chelating agent to remove the mercury. Mercury may remain in the body for long periods in some instances. However, it is believed that the emboli are converted slowly to metallic oxides and organic compounds of mercury and slowly eliminated primarily through the renal system [6] [8]. Those with large mercury deposits in accessible areas may benefit from surgical excision [7]. Those who develop renal failure often require hemodialysis [6].

\section{Conclusion}

Disseminated elemental mercury thromboembolism is a rare diagnosis to make with the emergency department complaint of chest pain. The chest radiograph will provide the provider with radiograph evidence of a radiopaque intravascular exposure that will require confirmation via blood and urine mercury levels. The patient's need for chelation therapy for removal of the mercury is based on clinical symptomatology and/or the mercury level on blood or urine toxicology screens. The amount of mercury detected does not have a direct relationship to clinical symptoms or end-organ damage. This patient was fortunate to have no long-term sequelae from his intentional parenteral mercury administration [4] [5]. 


\section{References}

[1] Shareeff, M., Yasser, B., Adabala, R. and Raoof, S. (2000) Shortness of Breath after Suicide Attempt. Chest, 118, 837838. http://dx.doi.org/10.1378/chest.118.3.837

[2] Chitkara, R., Seriff, N. and Kinas, H. (1978) Intravenous Self-Administration of Metallic Mercury in Attempted Suicide. Chest, 73, 234-236. http://dx.doi.org/10.1378/chest.73.2.234

[3] Kline, J.A., et al. (2008) Prospective Multicenter Evaluation of the Pulmonary Embolism Rule-Out Criteria. Journal of Thrombosis and Haemostasis, 6, 772-780. http://dx.doi.org/10.1111/j.1538-7836.2008.02944.x

[4] McFee, R.B. and Caraccio, T.R. (2001) Intravenous Mercury Injection and Ingestion: Clinical Manifestations and Management. Clinical Toxicology, 39, 733-738. http://dx.doi.org/10.1081/clt-100108515

[5] Clarkson, T.W., Magus, L. and Myers, G.J. (2003) The Toxicology of Mercury: Current Exposures and Clinical Manifestations. The New England Journal of Medicine, 349, 1731-1737. http://dx.doi.org/10.1056/NEJMra022471

[6] Deschamps, F., Strady, C., Deslee, G., Menciere-Faroy, B. and Deschamps, S. (2002) Five Years of Follow-Up after Elemental Mercury Self-Poisoning. The American Journal of Forensic Medicine and Pathology, 23, 170-172. http://dx.doi.org/10.1097/00000433-200206000-00012

[7] Tan, A., Neo, W.T. and Phua, J. (2010) An Element of Surprise. American Journal of Medicine, 123, 910-912. http://dx.doi.org/10.1016/j.amjmed.2010.06.007

[8] Vallant, B., Deutsch, J., Muntean, M. and Goessler, W. (2008) Intravenous Injection of Metallic Mercury: Case Report and Course of Mercury during Chelation Therapy with DMPS Intravenous Injection of Metallic Mercury. Clinical Toxicology, 46, 566-569. http://dx.doi.org/10.1080/15563650701725102 